\title{
Boundary conditions on the envelope function of convective rolls close to onset
}

\author{
M. C. Cross \\ Bell Laboratories, Murray Hill, New Jersey 07974
}

(Received 24 August 1981; accepted 12 March 1982)

Boundary conditions are derived for the envelope function of convective rolls approaching a rigid sidewall at an arbitrary orientation. This generalizes previous results for parallel and perpendicular rolls. It provides the first step in a study of the convective pattern to be expected close to onset in Rayleigh-Benard cells of any cross section, and allows consideration of more complicated patterns in rectangular or circular cross sections.

\section{INTRODUCTION}

Close to the onset of convection in laterally large Rayleigh-Benard cells the analysis of the hydrodynamic equations may be considerably simplified ${ }^{1.2}$ by the introduction of a slowly varying complex envelope function which describes the slow modulation of the roll pattern. An equation of motion (the "amplitude equation") for the envelope function may be derived from the hydrodynamic equations to give a complete description of the fluid away from the sidewalls. In addition boundary conditions on the envelope function must be specified so that the velocities and temperature satisfy the physical boundary conditions at the sidewalls. The amplitude equation plus boundary conditions provide an economical method for studying the influence of lateral boundaries on convection close to onset (for example see Refs. 3 and 4).

Segel $^{2}$ derived boundary conditions on the envelope function for two cases: rolls parallel to the sidewall; and rolls perpendicular to the sidewall. Later, Brown and Stewartson ${ }^{5}$ corrected the latter result. These boundary conditions suffice for convection in rectangular cells if the rolls are parallel to one or the other side. Although such patterns are observed, ${ }^{6}$ experiments in large boxes suggest other patterns may also be stable. ${ }^{7}$ Thus, to study more general states in rectangular cells, and also the patterns in cells of other cross sections, rolls at an arbitrary angle to the sidewall should also be considered.

Two questions must be answered. Firstly, may rolls approach a sidewall at an arbitrary angle, or are they in fact restricted to be parallel or perpendicular to the boundary? This is the question of the existence of boundary conditions on the direction of the normal to the rolls $\hat{n}$. Secondly, if the former result is found, what are the boundary conditions on the envelope function for arbitrary orientation? These questions will be answered in the present paper.

The application of these results to the analysis of the convection pattern in cylinders of arbitrary cross section will be deferred to a subsequent paper. However, to provide some motivation, consider the problem of the convection pattern in a circular cylinder, an important question in understanding recent experiments on turbulence close to onset. ${ }^{8}$ Certainly a possible arrangement is one of concentric rolls (which are therefore parallel to the boundaries) analyzed by
Brown and Stewartson ${ }^{3}$ and observed by Koschmieder and Pallas. ${ }^{9}$ Alternatively, if rolls may approach the sidewall at an arbitrary angle one might expect a pattern of parallel straight rolls. Such a pattern may have been observed as a stable, stationary state by Chen and Whitehead. ${ }^{10}$ Indeed, since the concentric pattern has a region of reduced amplitude convection near the center, ${ }^{3}$ an instability towards the liner pattern seems possible. ${ }^{11}$ On the other hand, if the rolls (if not parallel) must come in perpendicular to the walls, then severe distortions will be forced onto the pattern, probably involving dislocations in the rolls, making this alternative less favorable. These questions may be analyzed close to onset within the framework of the amplitude equation, once the boundary conditions are known.

\section{HYDRODYNAMIC EQUATIONS AND THE ENVELOPE FUNCTION}

The hydrodynamic equations used will be the Oberbeck-Boussinesq ${ }^{12}$ approximation to the full Navier-Stokes equations. They describe the motion under gravity $g$ of a fluid with density $\rho$, thermal diffusivity $\kappa$, kinematic viscosity $v$ and thermal expansion coefficient $\alpha$, and are equations for the horizontal velocity $\mathbf{u}=\left(u_{x}, u_{y}\right)$, the vertical velocity $w$, and $T$ the deviation of the temperature from the linear conducting profile:

$$
\begin{aligned}
\frac{\partial \mathbf{u}}{\partial t}= & \sigma\left(\nabla_{\perp}^{2}+\frac{\partial^{2}}{\partial z^{2}}\right) \mathbf{u}-\left(w \frac{\partial}{\partial z}+\mathbf{u} \cdot \nabla_{\perp}\right) \mathbf{u}_{1}-\nabla_{\perp} P, \\
\frac{\partial w}{\partial t}= & \sigma T+\sigma\left(\nabla_{\perp}^{2}+\frac{\partial^{2}}{\partial z^{2}}\right) w \\
& -\left(w \frac{\partial}{\partial z}+\mathbf{u} \cdot \nabla_{\perp}\right) w-\frac{\partial P}{\partial z}, \\
\frac{\partial T}{\partial t}= & R w+\left(\nabla_{1}^{2}+\frac{\partial^{2}}{\partial z^{2}}\right) T-\left(w \frac{\partial}{\partial z}+\mathbf{u} \cdot \nabla_{1}\right) T,
\end{aligned}
$$

together with the incompressibility condition:

$$
\boldsymbol{\nabla}_{\perp} \cdot \mathbf{u}+\frac{\partial w}{\partial z}=0
$$

Distance, time, and temperature are scaled by $d, d^{2} / k$, and $\kappa v / a g d^{3}$, respectively, where $d$ is the cell height. Also, $\sigma$ is the Prandtl number

$$
\sigma=v / \kappa,
$$


and $R$ is the Rayleigh number (the dimensionless form of the temperature difference across the cell that drives the fluid motion). The symbol $\nabla_{\perp}$ denotes the gradient in the horizontal plane.

The description of the system close to onset in terms of an envelope function may be understood as follows. At the critical Rayleigh number in a laterally infinite system, $R_{0}$, the hydrodynamic variables of the critical solution are given by

$$
(\mathbf{u}, w, T) \propto\left[\hat{n} u_{0}(z), w_{0}(z), T_{0}(z)\right] \exp \left(i q_{0} \hat{n} \cdot r_{1}\right)+\text { c.c. }
$$

where $u_{0}, w_{0}, T_{0}$ are found by solving the time independent linear equations, together with the boundary conditions at the upper and lower plates, and $q_{0}$ is the magnitude of the wave vector that minimizes $R_{0}(q)$. The unit vector $\hat{n}$ specifies the arbitrary direction of the rolls. Just above onset a more general state based on a single roll structure is

$$
\begin{aligned}
(\mathbf{u}, w, T)= & \epsilon^{1 / 2} A_{0}\left(X^{\prime}, Y^{\prime}, \tau\right)\left[\hat{n} u_{0}(z), w_{0}(z), T_{0}(z)\right] \\
& \times \exp \left(i q_{0} \hat{n} \cdot r_{1}\right)+\text { c.c. }+O(\epsilon),
\end{aligned}
$$

where $\epsilon=\left(R-R_{0}\right) / 18 \pi^{2}$ provides the small expansion parameter and $A_{0}$ is the complex envelope function that describes the slow modulation of the basic pattern. The $x^{\prime}$ direction is chosen along $\hat{n}$, and then $X^{\prime}, Y^{\prime}, \tau$ are coordinates scaled according to

$$
\begin{aligned}
& X^{\prime}=\epsilon^{1 / 2} x^{\prime}, \\
& Y^{\prime}=\epsilon^{1 / 4} y^{\prime} q_{0}{ }^{1 / 2}, \\
& \tau=\epsilon t\left(\xi_{0}^{2} / \tau_{0}\right),
\end{aligned}
$$

so that $A_{0}$ satisfies the amplitude equation in the form

$$
\frac{\partial A_{0}}{\partial \tau}=\left(1-\left|A_{0}\right|^{2}\right) A_{0}+\left(\frac{\partial}{\partial X^{\prime}}-\frac{i}{2} \frac{\partial^{2}}{\partial Y^{\prime 2}}\right)^{2} A_{0} \text {. }
$$

The form of Eq. $(8 \mathrm{c})$ is chosen to conform with previous work ${ }^{4,13}$ with parameters $\tau_{0}, \xi_{0} O(1)$ numbers, and the numerical factors in $u_{0}, w_{0}$, and $T_{0}$ are chosen to give unity for the nonlinear coupling coefficient in Eq. (9).

The amplitude equation (9) was derived by Newell and Whitehead ${ }^{1}$ and Segel ${ }^{2}$ assuming the mathematically convenient, but physically unrealistic, stress-free upper and lower boundary conditions

$$
\frac{\partial \mathbf{u}}{\partial z}=w=T=0 ; \quad z= \pm \frac{1}{2} .
$$

With this assumption the vertical and horizontal dependences separate, $\partial u_{0} / \partial z, w_{0}$, and $T_{0}$ are simply proportional to $\cos \pi z$, and the coefficients in the amplitude equation are easily calculated

$$
\tau_{0}^{-1}=\frac{3 \pi^{2} \sigma}{2(\sigma+1)} ; \quad \xi_{0}^{2}=\frac{8}{3 \pi^{2}} .
$$

The amplitude equation has also been derived ${ }^{13}$ for the physically relevant "rigid" boundary conditions. However to date no progress has been made on the lateral boundary conditions then to be imposed on the envelope function, and in this paper, following the previous workers, ${ }^{2,3}$ the simpler Eq. (10) will be assumed. It is not thought that imposing the rigid upper and lower boundary conditions will lead to qualitative changes in the results.

At the lateral sidewalls it is important to impose the physical boundary conditions. These are zero velocities:

$$
u_{x}=u_{y}=w=0 \text {, }
$$

and for the temperature

$$
T+\mu \frac{\partial T}{\partial x}=0,
$$

where $\mu$ is a constant depending on the thermal conduction of the sidewalls. ${ }^{4}$ Equation (13) implies a sharp onset at a critical Rayleigh number $R_{c}$, with zero velocities below $R_{c}$, and may be considered as a rather general ideal thermal boundary condition.

The correct scheme for deriving boundary conditions on the envelope function was put forward by Brown and Stewartson. ${ }^{5}$ They noted that it is incorrect simply to substitute the expressions Eq. (7) into Eqs. (12) and (13), for the problem then becomes overdetermined. Instead, it is necessary to allow for the excitation of additional solutions localized near the walls. The problem is tractable, however, because near the rigid sidewall the fluid velocities are small, and the solution here, including the rapidly varying walllocalized part, is correctly given by the linearized hydrodynamic equations. Introducing the coordinate $X$, which is the distance $x$ from the wall suitably scaled according to Eq. (8), then the boundary condition on the envelope function $A_{0}(X \rightarrow 0)$ is found by matching to the solutions to the linearized hydrodynamic equations for $x>1$, where the wall-localized solutions are negligible and Eq. (7) is correct. This is the procedure used by Brown and Stewartson ${ }^{5}$ for rolls parallel or perpendicular to the sidewall. Here it is generalized to rolls at an arbitrary orientation.

\section{DERIVATION OF THE BOUNDARY CONDITIONS}

The derivation consists of two parts; solving the linearized hydrodynamic equations near the sidewall, and then matching the solution for $x \rightarrow \infty$ to the solution given by the envelope function for $X \rightarrow 0$.

\section{A. Linearized hydrodynamic equations}

It is sufficient to solve the equations for $R=R_{0}$; for $x<\epsilon^{-1 / 2}$ where the solutions are required, this introduces negligible error. ${ }^{5}$ The equations are conveniently solved by introducing auxiliary variables $v\left(\mathbf{r}_{1}, z\right)$ and $\xi\left(\mathbf{r}_{1}, z\right)$ satisfying

$$
\begin{aligned}
& \left(\nabla_{\perp}^{2}+\frac{\partial^{2}}{\partial z^{2}}\right)^{3} v-R_{0} \nabla_{\perp}^{2} v=0, \\
& \frac{\partial}{\partial z}\left(\nabla_{\perp}^{2}+\frac{\partial^{2}}{\partial z^{2}}\right) \xi=0,
\end{aligned}
$$

in terms of which the solutions to the linearized hydrodynamic equations are ${ }^{14}$

$$
\begin{aligned}
& w=-\nabla_{\perp}^{2} v, \\
& \mathbf{u}=\nabla_{\perp} \frac{\partial}{\partial z} v+\operatorname{curl}_{\perp} \zeta, \\
& T=\left(\nabla_{\perp}^{2}+\frac{\partial^{2}}{\partial z^{2}}\right)^{2} v .
\end{aligned}
$$

Note that $\zeta$ determines the vertical vorticity

$$
\frac{\partial u_{y}}{\partial x}-\frac{\partial u_{x}}{\partial y}=-\nabla_{1}^{2} \zeta
$$


which in general decays away from the sidewalls (except for a $z$-independent component which corresponds to a uniform rotation and is not relevant in this work), but may be excited near the walls.

The solution to Eqs. (14) and (15) satisfying the boundary conditions Eq. (10) at stress-free top and bottom boundaries takes the form

$$
\begin{aligned}
v= & \sum_{q_{y}}\left[\left(a_{+}+b_{+} x\right) \exp \left(i q_{x} x\right)\right. \\
& \left.+\left(a_{-}+b_{-} x\right) \exp \left(-i q_{x} x\right)+c \exp (-\lambda x)\right] \\
& \times \exp \left(i q_{y} y\right) \cos \pi z, \\
\zeta= & \sum_{q_{y}} a_{0} \exp (-\bar{\lambda} x) \exp \left(i q_{y} y\right) \sin \pi z,
\end{aligned}
$$

where the sidewall is at $x=0$ and additional solutions increasing exponentially with $x$ have been left out in anticipation of the matching to be performed for $x>1$. The Fourier expansion in $q_{y}$ allows arbitrary variation along the wall, and for each $q_{y}$ the value of $q_{x}$ is given by

$$
q_{x}^{2}+q_{y}^{2}=q_{0}^{2}=\frac{1}{2} \pi^{2}
$$

and then

$$
\begin{aligned}
& \lambda^{2}=9 \pi^{2} / 2-q_{x}^{2}, \\
& \bar{\lambda}^{2}=3 \pi^{2} / 2-q_{x}^{2} .
\end{aligned}
$$

All $a_{ \pm}, b_{ \pm}, a_{0}$ and $c$ are functions of $q_{y}$, and reality conditions give relationships such as

$$
a_{+}\left(q_{y}\right)=a_{-}^{*}\left(-q_{y}\right) .
$$

Equations (18) and (19) are the general solution once the $z$ dependence for matching to Eq. (7) is assumed.

The unknown coefficients in Eqs. (18) and (19) may be related by applying the boundary conditions Eqs. (12) and (13) and using Eq. (16). Since the equations and boundary conditions are linear, each $q_{y}$ component may be treated separately. Satisfying the boundary conditions for all $y$ then leads, in general, to four linear homogeneous complex equations for the six complex unknowns $a_{ \pm}, b_{ \pm}, a_{0}, c$. The explicit equations are derived in Appendix A. From these six equations $a_{0}$ and $c$ may be eliminated to given two linear complex equations for the four complex unknowns of interest $a_{ \pm}, b_{ \pm}$that, on matching, will provide boundary conditions on the envelope function:

$$
M_{24}\left(a_{+}, b_{+}, a_{-}, b_{-}\right)=0,
$$

with $\mathrm{M}_{24}$ a $2 \times 4$ matrix with $O(1)$ complex coefficients that depend on $q_{y}$. For most of the ensuing arguments it is sufficient to know the number of linear relationships, together with the fact that for general $q_{y}$ the coefficients in these relationships are $O(1)$. The detailed form of the coefficients is not needed.

\section{B. Matching to the envelope function}

We will suppose that the envelope function describes a set of rolls approaching a plane boundary with the roll nor$\mathrm{mal} \hat{n}$ at an angle $\theta$ to the surface normal $\hat{s}$. For simplicity we assume variation only with the $X$ coordinate (normal to the wall), although the same results would apply with variation along the wall and in time on the slow scales $Y$ and $\tau$ in addition. With these assumptions the envelope function satisfies

$$
\left(\cos ^{2} \theta \frac{\partial^{2}}{\partial X^{2}}+1-\left|A_{0}\right|^{2}\right) A_{0}=0,
$$

since, for $\theta$ not to close to $\frac{1}{2} \pi$, the fourth-order derivative terms implied by Eq. (9) are of relative order $\epsilon^{1 / 2}$ and are negligible. The hydrodynamic variables are then given by

$$
v=\epsilon^{1 / 2}\left[A_{0}(X) \exp \left(i \mathbf{q} \cdot \mathbf{r}_{1}\right)+\text { c.c. }\right] \cos \pi z,
$$

with

$$
\mathbf{q}=q_{0}(\cos \theta, \sin \theta),
$$

which must be matched for $X \rightarrow 0$ with the solutions Eqs. (18) and (19) for $x>1$.

A naive matching of the two equations leads to a surprising result: for $q_{y}$ equal to $q_{0} \sin \theta$ the coefficients $a_{-}$and $b_{-}$are identically zero, and Eq. (22) becomes a system of two linear homogeneous equations in two unknowns $a_{+}, b_{+}$that in general will have no nonzero solution. A similar conclusion arises for the negative value of $q_{y}$, and all other $q_{y}$ components are absent. This argument implies that for general roll orientations, the assumption of a single roll pattern as $X \rightarrow 0$ is inconsistent with the physical boundary conditions. The matching may, however, be achieved if in addition a "conjugate" set of rolls is assumed present near the boundary, based on a wave vector $q_{0}(-\cos \theta, \sin \theta)$, so that $a_{-}$ and $b_{-}$are nonzero. Since a pattern consisting of the superposition of two sets of rolls is unstable away from boundaries, ${ }^{14}$ these conjugate rolls would be expected to decay away from the boundaries.

More careful investigation shows that there does in fact exist a nontrivial solution at $O\left(\epsilon^{1 / 2}\right)$ satisfying Eq. (22), since matching gives, for $q_{y}=q_{0} \sin \theta$,

$$
\begin{aligned}
& a_{+}=\epsilon^{1 / 2} A_{0}(0), \\
& b_{+}=\epsilon \frac{\partial A_{0}}{\partial X}=O(\epsilon) .
\end{aligned}
$$

Thus Eq. (22) is satisfied at $O\left(\epsilon^{1 / 2}\right)$ by taking

$$
A_{0}(0)=0, \quad \frac{\partial A_{0}}{\partial X}=O(1)
$$

for then all $a_{ \pm}, b_{ \pm}$are zero at this order and Eq. (27) then provides sufficient boundary conditions for nontrivial solutions of the amplitude equation (23). However at $O(\epsilon)$ Eq. (22) cannot be satisfied with the assumption of a single set of rolls, and it is at this order that the conjugate rolls at an angle $-\theta$ must be excited. To show that Eqs. (27) and (23) are then sufficient to determine the envelope function away from the sidewalls, it is necessary to prove that these conjugate rolls may indeed decrease away from the boundary, so that Eq. (23) for $A_{0}$ and Eq. (24) for the hydrodynamic variables are not significantly changed. The rather intricate argument for this is deferred to Appendix $B$. The conclusion found there is that the conjugate rolls are indeed excited at $O(\epsilon)$, but may be chosen to decay away from the sidewall (for $X \gtrsim 1$ ). Equations (23) and (27) are then sufficient to determine the lowestorder envelope function $A_{0}$, and at this order the conjugate 
rolls need not be explicitly considered. If an analysis to higher order is attempted (e.g., along the lines of Ref. 4), the boundary conditions on the higher-order approximations to the envelope function will be altered.

It is interesting to note that the boundary condition $\mathrm{Eq}$. (27) for general orientations is, at this lowest order, the same as that found previously for rolls parallel to the sidewall. On the other hand, for rolls perpendicular to the sidewall the conditions are quite different. The way the result for general orientation tends to these special limits is considered in Appendix C. It is found that the analysis leading to Eq. (27) breaks down for $|\pi / 2-\theta| \leqslant \epsilon^{1 / 4}$ (rolls close to perpendicular.) This is to be expected, since it is in this limit that the fourth-order gradient terms must be included in the amplitude equation (23).

\section{CONCLUSIONS}

The main result of this paper is that convective rolls close to onset can indeed approach a boundary at an arbitrary angle, although, in general, small amplitude conjugate rolls will also be excited. The boundary condition on the lowest-order envelope function is the same as for rolls parallel to the sidewall (the case of nearly normal rolls $|\pi / 2-\theta| \leqslant \epsilon^{1 / 4}$ being special), with modifications at the next order.

This paper has only addressed the question of conditions forced on the envelope function by the physical boundary conditions. We have found that the orientation of the rolls is not fixed by the physical boundary conditions. A more difficult question is the possibility of orienting effects given by solving the amplitude equation subject to the boundary conditions derived here. For this question the boundary region cannot be studied in isolation; methods treating the solution over the whole cell must be developed. This will be considered elsewhere. [The separation of these two questions is made possible close to onset by the presence of the long length scale, usually $O\left(\epsilon^{-1 / 2}\right)$, that governs the variation of the envelope function. For $\epsilon$ of order unity such a separation is not possible, and a completely new approach would be needed.]

As yet there seem to be no experimental confirmation of the presence of the conjugate rolls predicted here. This is not too surprising, since where the analysis is valid the conjugate rolls are expected to be of small amplitude, and will manifest themselves in a flow visualization experiment merely as a small perturbation of the basic rolls close to the boundary. Also, in many experiments to date the rolls are found to approach the boundaries perpendicularly, a configuration in which conjugate rolls are not excited, and $\epsilon$ is $O(1)$, so that the analysis presented here is not valid. To test this particular prediction, more quantitative measurements close to the boundaries and with $\epsilon<1$ would be needed.

Note added in proof: Since this work was done, Siggia and $\mathrm{Zippelius}{ }^{15}$ have shown that for the mathematically convenient model of free-slip boundaries, an additional term must be added to the amplitude equation (9) due to a nonlinear coupling to the long wavelength, vertically uniform, vorticity mode. However the physical boundary conditions are linear, and the boundary conditions on the envelope function are found from the components varying as $\sin \pi z$ or $\cos \pi z$, and so these results should remain unchanged. It is conceivable, however, that the uniform vertical vorticity may distort the conjugate rolls. Since the vertical vorticity mode is suppressed by rigid upper and lower boundaries we may hope that all the results calculated here are qualitatively correct for this experimentally relevant case. An infinite Prandlt number plays a similar role with free-slip boundaries, and the conclusions are strictly correct in this limit.

\section{APPENDIX A: BOUNDARY RELATIONS}

The physical boundary conditions Eqs. (12) and (13) are to be applied in turn to Eq. (18), using Eqs. (16), at $x=0$ and for all $y$. This gives four complex equations

$$
\begin{aligned}
& i q_{x}\left(a_{+}-a_{-}\right)+\left(b_{+}+b_{-}\right)-\lambda c-i q_{y} a_{0} / \pi=0, \\
& i q_{y}\left(a_{+}+a_{-}\right)+i q_{y} c-\bar{\lambda} a_{0} / \pi=0, \\
& -\frac{1}{2} \pi^{2}\left(a_{+}+a_{-}\right)+2 i q_{x}\left(b_{+}-b_{-}\right)+4 \pi^{2} c=0, \\
& {\left[3 \pi^{2}\left(a_{+}+a_{-}\right)-8 i q_{x}\left(b_{+}-b_{-}\right)+12 \pi^{2} c\right]} \\
& \quad+\mu\left[3 \pi^{2} i q_{x}\left(a_{+}-a_{-}\right)\right. \\
& \left.\quad+\left(8 q_{x}^{2}+3 \pi^{2}\right)\left(b_{+}+b_{-}\right)-12 \pi^{2} \lambda c\right]=0 .
\end{aligned}
$$

Although it is possible to continue with the general analysis, it seems sufficient to note that putting $a_{-}$and $b_{-}$to zero would leave four linear homogeneous equations in four unknowns $\left(a_{+}, b_{+}, c, a_{0}\right)$ which will have only the trivial zero solution, except possibly for certain special orientations $q_{x} / q_{y}$. We will consider further the special case $\mu=0$ (corresponding to sidewalls that are good conductors) to illustrate these conclusions.

For the case $\mu=0$, eliminating the uninteresting variables $c$ and $a_{0}$ leads to the two equations:

$$
\begin{aligned}
& 9 \pi^{2}\left(a_{+}+a_{-}\right)-28 i q_{x}\left(b_{+}-b_{-}\right)=0, \\
& 28 i q_{x}\left(a_{+}-a_{-}\right)+\left(27 q_{y}^{2} / \bar{\lambda}+\lambda\right)\left(a_{+}+a_{-}\right) \\
& \quad+28\left(b_{+}+b_{-}\right)=0 .
\end{aligned}
$$

If we put $a_{-}, b_{-}$to zero at all orders in $\epsilon$ (i.e., no conjugate rolls), it is easy to check that there are no nonzero solutions for $a_{+}, b_{+}$for any orientation of the rolls. Following the analysis of Appendix B we must allow nonzero $a_{-}$at $O(\epsilon)$, but may put $b_{-}$to zero at this order. Then, eliminating $a_{-}$ leads to a relationship between the variables $a_{+}$and $b_{+}$that will match to the envelope function of the fundamental rolls

$$
18 \pi^{2} i q_{x} a_{+}+\left[\left(9 \pi^{2}+28 q_{x}^{2}\right)+i q_{x}\left(\lambda+27 q_{y}^{2} / \bar{\lambda}\right)\right] b_{+}=0 .
$$

The identities

$$
\begin{aligned}
& a_{+}=\epsilon^{1 / 2} A_{0}(0)+\epsilon A_{1}(0)+\ldots, \\
& b_{+}=\epsilon A^{\prime}{ }_{0}(0)+\ldots,
\end{aligned}
$$

where $A_{1}$ is the next higher-order approximation to the envelope function (see Appendix B) then lead to the boundary conditions for rolls at a general angle: 


$$
A_{0}(0)=0 \text {, }
$$

$$
\begin{aligned}
& 18 \pi^{2} i q_{x} A_{1}(0)+\left[\left(9 \pi^{2}+28 q_{x}^{2}\right)\right. \\
& \left.+i q_{x}\left(\lambda+27 q_{y}^{2} / \bar{\lambda}\right)\right] A_{0}^{\prime}(0)=0 .
\end{aligned}
$$

\section{APPENDIX B: EXCITATION OF THE CONJUGATE ROLLS}

Extending the definition of the envelope function to higher order (e.g., see Ref. 4), and including the conjugate rolls leads to the expression for $v$ away from the sidewalls:

$$
\begin{aligned}
v= & \epsilon^{1 / 2}\left\{\left[A_{0}(X)+\epsilon^{1 / 2} A_{1}(X)\right] \exp \left[i q_{0}(x \cos \theta+y \sin \theta)\right]\right. \\
& +\epsilon^{1 / 2} B_{1}(X) \exp \left[i q_{0}(-x \cos \theta+y \sin \theta)\right] \\
& + \text { c.c. }\} \cos \pi z+O(\epsilon) \cos 2 \pi z+O\left(\epsilon^{3 / 2}\right),
\end{aligned}
$$

where $B_{0}(X)$ is assumed to be zero, and a solution is sought for $B_{1}(X)$ that does not grow from $O(1)$ away from the boundary, thereby invalidating the expansion scheme.

The equation for $A_{0}$, found by expanding the hydrodynamic equations to $O\left(\epsilon^{3 / 2}\right)$, is unchanged

$$
\left(\cos ^{2} \theta \frac{\partial^{2}}{\partial X^{2}}+1-\left|A_{0}\right|^{2}\right) A_{0}(0)=0 ; \quad A_{0}(0)=0
$$

while at $O\left(\epsilon^{2}\right)$ we find the equation for $B_{1}$ :

$$
\left(\cos ^{2} \theta \frac{\partial^{2}}{\partial X^{2}}+1-(1+\beta)\left|A_{0}\right|^{2}\right) B_{1}=0,
$$

with $\beta$ a positive coefficient depending on $\theta$ which may be found from Refs. 1 and 13, together with an equation for $A_{1}$ that we will not need. Equation (B2) may readily be solved:

$$
A_{0}=\tanh (X / \sqrt{2} \cos \theta) .
$$

Substituting this expression into Eq. (B3) then leads to a complicated equation for $B_{1}(X)$, which is however linear in $B_{1}$. For large $X$ the asymptotic dependence is

$$
B_{1} \sim \exp \left( \pm \beta^{1 / 2} X\right) \text {. }
$$

Since we require the decaying solution, integrating back from large $X$ will give a relationship

$$
B_{1}(0)=\left.\gamma \frac{\partial B_{1}}{\partial X}\right|_{x=0},
$$

with $\gamma(\theta, \beta)$ an $O(1)$ number. Finally, matching Eq. (B1) for $X \rightarrow 0$ to Eqs. (18) for $x>1$ gives

$$
\begin{array}{ll}
a_{+}=\epsilon A_{1}(0), & b_{+}=\epsilon A_{0}^{\prime}(0), \\
a_{-}=\epsilon B_{1}(0), & b_{-}=0+O\left(\epsilon^{3 / 2}\right),
\end{array}
$$

so that, with the inclusion of the conjugate rolls at $O(\epsilon) \mathrm{Eq}$. (22) may be satisfied. In fact, $a_{-}$may then be eliminated to give a single boundary condition (depending on $\theta$ ) relating $A_{1}(0)$ to $A_{0}^{\prime}(0)$, as in the case ${ }^{4}$ of $\theta=0$ (Appendix A).

\section{APPENDIX C: SPECIAL CASES}

It is of some interest to consider the special cases $\theta=0$ or $\pi / 2$, studied previously, in the same framework as used here for general angles.

\section{Parallel rolls, $\theta=\pi / 2$}

For parallel rolls, $q_{y}$ is zero and Eq. (18) becomes

$$
\begin{aligned}
v= & {\left[(a+b x) \exp \left(i q_{0} x\right)+\left(a^{*}+b^{*} x\right) \exp \left(-i q_{0} x\right)\right.} \\
& +c \exp (-\lambda x)] \cos \pi z,
\end{aligned}
$$

with $c$ now a real number, and $\zeta$ is zero. The hydrodynamic boundary conditions consist of three real equations for the five real unknows: $\operatorname{Re} a, \operatorname{Im} a, \operatorname{Re} b, \operatorname{Im} b, c$. These may in general be reduced to one complex equation

$$
a+\alpha_{-} b+\beta_{-} b^{*}=0,
$$

with $\alpha_{-}, \beta_{-} 0(1)$ complex constants. Using the same rotation as in Appendix B, Eq. (C2) gives the boundary conditions

$$
\begin{aligned}
& A_{0}(0)=0, \\
& A_{1}(0)+\alpha_{-} A_{0}^{\prime}(0)+\beta_{-} A_{0}^{\prime *}(0)=0,
\end{aligned}
$$

as found previously. ${ }^{2,3,4}$ Continuity with the result at general $\theta$ is shown by allowing some phase modulation

$$
A_{0}(X, Y)=A_{0}(X) \exp (i Q Y)
$$

effectively rotating the rolls slightly away from parallel. Applying Eq. (16) at $X=0$ for each $Y$ shows the excitation of a component to $A_{1}$ varying as $\exp (-i Q Y)$, that is just the amplitude of the conjugate rolls.

\section{Perpendicular rolls, $\theta=0$}

In this case the solution to the linearized hydrodynamic equations close to the sidewall becomes ${ }^{5}$

$$
\begin{aligned}
v= & {\left[a+b x+f x^{2}+g x^{3}+c \exp (-\lambda x)\right] \exp \left(i q_{0} y\right) } \\
& \times \cos \pi z+\text { c.c. } \\
\zeta= & {\left[a_{0} \exp (-\bar{\lambda} x) \exp \left(i q_{0} y\right)+\text { c.c. }\right] \sin \pi z }
\end{aligned}
$$

where we have anticipated the matching to $\bar{A}_{0}(X)$, independent of $Y$, defined by

$$
v=\epsilon^{1 / 2}\left[\bar{A}_{0}(X) \exp \left(i q_{0} y\right)+\text { c.c. }\right] \cos \pi z,
$$

away from the sidewall. Matching leads to the boundary conditions $^{5}$

$$
\bar{A}_{0}(0)=\bar{A}_{0}^{\prime}(0)=0 \text {, }
$$

Since Eq. (C8) takes on a quite different form than the general $\theta$ result, the limit $\theta \rightarrow \pi / 2$ of the analysis in Appendix $B$ should be studied in more detail. Such an analysis leads to the results

$$
a_{+}, a_{-}=O\left(\epsilon / \theta^{\prime 2}\right),
$$

but

$$
a_{+}+a_{-}=O\left(\epsilon / \theta^{\prime}\right),
$$

and

$$
b_{+}=O\left(\epsilon / \theta^{\prime}\right), b_{-}=O\left(\epsilon^{3 / 2} / \theta^{\prime 3}\right),
$$

where $\theta^{\prime}=\pi / 2-\theta$. Clearly, the analysis in Appendix B breaks down for $\theta^{\prime} \leqslant \epsilon^{1 / 4}$ when $b_{+}$and $b_{-}$become of comparable magnitude, and the conjugate rolls become of the same strength as the principal rolls. This is just the limit that can be described in terms of an envelope function based on perpendicular rolls, leading to Eq. (C8). 
(1978).

${ }^{4}$ M. C. Cross, P. G. Daniels, P. C. Hohenberg, and E. D. Siggia, Phys. Rev. Lett. 45, 898 (1980).

${ }^{5}$ S. N. Brown and K. Stewartson, Stud. in Appl. Math. 57, 187 (1977). See also P. G. Daniels, Proc. R. Soc. London Ser. A 358, 173 (1977).

${ }^{6} \mathrm{P}$. Berǵge and M. Dubois, Phys. Rev. Lett. 32, 1041 (1974).

${ }^{7} \mathrm{~J}$. P. Gollub (private communication).

${ }^{8}$ G. A.hlers and R. P. Behringer, Phys. Rev. Lett. 40, 712 (1978).

${ }^{9}$ E. L. Koschmieder and S. G. Pallas, Int. J. Heat Mass Transfer 17, 991
(1974).

${ }^{10}$ M. M. Chen and J. A. Whitehead, J. Fluid Mech. 31, 1 (1968).

${ }^{11}$ G. Ahlers, M. C. Cross, P. C. Hohenberg, and S. Safran, J. Fluid Mech. 110, 297 (1981)

${ }^{12}$ S. Chandrasekhar, Hydrodynamic and Hydromagnetic Stability (Oxford University Press, Oxford, 1968).

${ }^{13}$ M. C. Cross, Phys. Fluids 23, 1727 (1980).

${ }^{14}$ A. Schluter, D. Lortz, and F. Busse, J. Fluid Mech. 23, 129 (1965)

${ }^{15}$ E. Siggia and A. Zippelius, Phys. Rev. Lett. 47, 835 (1981). 